\title{
Editorial
}

\section{The importance of an Evidence-Based Approach}

Jason A. Griggs*

As a professor of dental materials science, I am sometimes asked to provide a ranking of current restorative materials that have the best performance for each application. Those of you engaged in research in this area will eventually have the same requested of you. However, attempts to honor such requests have short-lived benefit and actually perform a disservice to the clinicians who are requesting our advice. Every year, I tell the new students at my school that a list of approved materials and devices would become obsolete within two years of their graduation. It is much more useful to provide students with a foundation of basic materials science knowledge and to teach them the critical thinking skills that they will use to evaluate future advertisements, bench tests, clinical trials, and meta-analyses and to weight those sources of information appropriately in making their own decisions about which clinical protocols to adopt. This sort of evidence-based dentistry was finally added to coursework at my school four years ago, and I hope that it will be adopted soon by all dental schools. The preclinical materials science instruction is limited to laboratory exercises in many dental schools. However, clinicians must have more than laboratory knowledge to apply the critical thinking skills of evidence-based dentistry. They need a firm foundation of the fundamental forces regulating the behavior of materials at the most basic level atomic bonding, dislocation motion, and phase transformations. The founding professors of the curriculum at my school, Dr. Lyle Zardiackas and Dr. Stephen Bayne, shared this vision, and I have continued their emphasis. This is why students at the University of Mississippi School of Dentistry benefit from 34 hours of basic materials science before they begin to study dental materials. They take the materials science course concurrently with a course on evidence-based dentistry, in which they learn how to critique and classify different types of publications. We will find out in the near future how this course combination serves them as they embark into private practice.

As a researcher, I have had the pleasure of serving on several Small Business Innovation Research grant review panels and of serving for six years on the NIH Oral, Dental, and Craniofacial Sciences (ODCS) study section. It was an exciting and educational time for me as I was assigned to read 24 grant applications per year on dental materials and to listen to debates regarding the possible funding or rejection of over 200 grant applications per year in other areas of dentistry. This engineer suddenly learned a lot of biology! However, my primary source of enthusiasm was the good feeling that I was doing my civic duty in deciding how billions of dollars and a large workforce of research staff would best be allocated. My enthusiasm waned as the current depressed economy took hold and the government budget was reduced to a size that could only fund a few projects per year. So many excellently designed projects were going without funding! I can imagine that the current situation is similar in Brazil. Now that funding agencies find themselves on a lean budget, it seems that only projects aimed developing new products or innovative device designs are receiving much consideration, and I find this trend disturbing. It robs us of the data that we need to perform evidence-based dentistry. The world's governments need to fund failure analysis and long-term clinical studies because those projects are less likely to be funded by materials manufacturers, who are already focused on product innovation and in vitro studies. The late Dr. Janet Quinn established a Practice-Based Research Network (PBRN) to retrieve failed prostheses and clinical notes from dentists for use in constructing databases of failure modes. Another such PBRN, Fracto Forum International 
(fractography.org), was established by Dr. Susanne Scherrer and Dr. Ulrich Lohbauer and is currently in its third year of activity. These networks are essential to troubleshooting our products and practices. Many of the product innovations that I reviewed as a member of the ODCS study section had promising in vitro data that did not translate to clinical success. If the practice of dentistry is to advance based on evidence of clinical performance instead of clever marketing, then academics and governments need to support failure analysis and the teaching of evidence-based dentistry.

The mindset that comes to dentists and engineers through application of the evidence-based approach is also useful outside of our professional roles. Those in our line of work are likely to be among the most educated members of their communities. We are called upon to serve on boards of directors for the local schools and churches and for city government. We serve as opinion leaders for the populace when scientific issues such as global warming, childhood vaccination, genetically modified organisms, and the origin of species are discussed. We need to inject a healthy level of skepticism among our fellow citizens to make them resistant to charlatans who prey upon ignorance and superstition for profit at the expense of our environment, educational systems, and justice systems. This can only be done by us as scientists who our local citizens know personally and trust. It is our duty as scientific professionals to teach an evidence-based approach to our students, our funding agencies, and our fellow citizens. 